\title{
Preparation and Characterization of Block Copolymers of Ordinary and Deuterated Styrenes
}

\author{
Yuhshuh Matsushita, Hiroyuki Furuhashi, Haruhisa CHOSHI, \\ Ichiro NoDA, Mitsuru Nagasawa, Teruo Fujimoto, ${ }^{*}$ \\ and Charles C. HAN** \\ Department of Synthetic Chemistry, Nagoya University, \\ Furo-cho, Chikusa-ku,.Nagoya 464, Japan. \\ *Department of Material Science and Technology, Technological University of Nagaoka, \\ Kamitomioka-cho, Nagaoka, Niigata 949-54, Japan. \\ **Characterization and Standards Group, Polymer Science and Standards Division, \\ National Bureau of Standards, United States Department of Commerce, \\ Washington D.C. 20234, U.S.A.
}

(Received October 13, 1981)

KEY WORDS Block Copolymer / Neutron Scattering / Deuterated Styrene / Narrow Molecular Weight Distribution / Anionic Polymerization /

Polymers containing deuterated segments, such as the block copolymers of ordinary and deuterated styrenes, are in demand for use in small angle neutron scattering techniques in polymer science. ${ }^{1-3}$ The samples should have narrow distributions with respect to both molecular weight and composition. However, the deuterated polymer samples used so far in neutron scattering measurements appear to have distributions broader than ordinary hydrogen polymer samples. ${ }^{4-6}$ In this work, an attempt was made to obtain polystyrenes containing a styrene- $d_{8}$ sequence as a block by the anionic polymerization technique, but the preparation of di-block and tri-block copolymers of styrene and styrene- $d_{8}$ was found to be much more difficult than that of ordinary block copolymers. The reason for this stemed from the particular kind of impurities contained in commercially available styrene$d_{8} .{ }^{* 1}$ It seems worth-while to report in detail of our method of preparation, since no deuterated styrene block copolymers of sufficiently narrow molecular weight distribution has as yet been reported in the literature.

\section{EXPERIMENTAL}

\section{Purification of Styrene- $d_{8}$ Monomer}

Styrene- $d_{8} \quad(98+$ atom $\% \mathrm{D})$ monomer was obtained from Aldrich Chemical Company. It was dried over calcium hydride, preliminarily distilled, and purified according to the methods described in RESUlts AND Discussion. A Toyo-Soda HLC-827 high speed liquid chromatograph equipped with high-resolution columns $(\mathrm{G} 2000 \mathrm{HG} 8 \times 2)$ was used for the purification. The monomer was dissolved in chloroform at $3.5 \mathrm{vol} \%$. The sampling was carried out every $25 \mathrm{~min}$ with a sampling volume of $6 \mathrm{ml}$. The elution time was about $50 \mathrm{~min}$. The elution curve of the monomer and impurities was monitored using both a refractometer and a U.V. detector. The purity of the monomer was examined with a Shimadzu GC-7A gas chromatograph provided with a high-resolution glass capillary column OV-101 SCOT (o.d. $0.9 \mathrm{~mm}$, i.d. 0.3 $\mathrm{mm}, 50 \mathrm{~m}$ long). Neutron activation analysis ${ }^{7}$ of the monomer was made at the National Bureau of Standards by a computerized system with a $\mathrm{Ge}(\mathrm{Li})$

*1 Disclaimer: Certain commercial materials and instruments are identified in this paper in order to specify the experimental procedure. In no case does such identification imply recommendation or endorcement by the National Bureau of Standards. 
detector applicable to a range of gamma-ray energies.

\section{Preparation of Block Copolymers}

Block copolymerization was carried out using the sequential monomer addition technique of the anionic polymerization method with $s$-butyllithium in benzene. The polymerization temperature was $35^{\circ} \mathrm{C}$ for both ordinary and deuterated monomers. For characterization of the polymeric intermediates in block copolymers, precursors were sealed off at each stage.

\section{Characterization of Block Copolymers}

The number- and weight-average molecular weights of the block copolymers and their precursors were measured in toluene at $25^{\circ} \mathrm{C}$, using a Hewlett-Packard Type 502 high speed membrane osmometer and a Fica automatic light scattering photometer, respectively. The molecular weight heterogeneity was examined by ultracentrifugation with a Beckman Spinco Model E ultracentrifuge in cyclohexane at $35^{\circ} \mathrm{C}$ and also on GPC chromatograms of Toyo-Soda HLC-827 equipped with high-resolution columns $(\mathrm{GMH} 6 \times 2)$. The mobile phase was THF. The GPC apparatus was calibrated with a series of Toyo-Soda standard polystyrenes.

The precipitational fractionation was made by changing the temperature of the cyclohexane solutions. Pyrolysis-gas chromatography (PGC) was carried out so as to analyze the content of the deuterated blocks in the samples prior to and following fractionation. The details of the PGC study are reported elsewhere. ${ }^{8}$

\section{RESULTS AND DISCUSSION}

The gas chromatogram of the monomer supplied by the manufacturer showed one and two peaks on the higher and lower boiling point sides of the main peak, respectively, as shown in Figure 1a. By comparing this with the chromatograms of styrene containing halogenated aromatic compounds, we identified the impurity on the higher boiling point side as monobromobenzene- $d_{5}$. Moreover, the neutron activation analysis detected the amount of bromide which was found to be in good agreement with the value calculated assuming that the impurity was monobromobenzene- $d_{5}$. For example, about 70 $\mu \mathrm{gg}^{-1}$ was found by neutron activation analysis and $50 \mu \mathrm{g} \mathrm{g}^{-1}$ by gas chromatography for a certain sample of styrene- $d_{8}$ monomer. Since the impurities of the lower side were found to be inactive for anionic active centers, they were not clearly identified but seemed to be alkyl benzenes on the basis of their elution times.

When benzophenone-sodium (BP-Na) ${ }^{9}$ was added to the deuterated monomer containing impurities, the characteristic dark-green color of BP-Na in the monomer gradually changed to a yellowgreen, indicating that the reaction of BP-Na with the impurities proceeded very slowly. It was confirmed by gas chromatography that the impurity on the higher boiling point side considerably decreased by the reaction but remained detectable even after
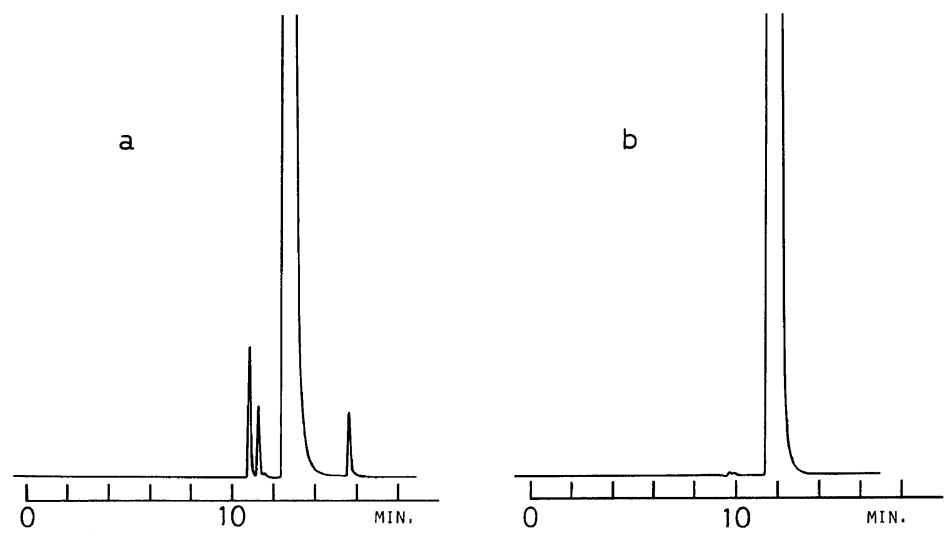

Figure 1. Gas chromatograms of styrene- $d_{8}$ monomer. (a) Before purification. The impurity content is of the order of $0.1 \mathrm{vol} \%$. (b) After purification. 
Block Copolymers of Ordinary and Deuterated Styrenes

Table I. Polymerization results for block copolymers before fractionation

\begin{tabular}{|c|c|c|c|c|c|}
\hline \multirow{2}{*}{$\begin{array}{l}\text { Sample } \\
\text { code }\end{array}$} & \multirow{2}{*}{$\frac{\text { Conversion }}{\%}$} & \multicolumn{3}{|c|}{$M_{n} \times 10^{-4}$ at various stages of copolymerization } & \multirow{2}{*}{$\frac{C_{\mathrm{d}}^{\mathrm{b}}}{\%}$} \\
\hline & & $\begin{array}{c}\text { First } \\
\text { precursors }\end{array}$ & $\begin{array}{l}\text { Second } \\
\text { precursor }\end{array}$ & $\begin{array}{c}\text { Final } \\
\text { polymers }\end{array}$ & \\
\hline TUN-14 & 100 & $3.3(2.7)^{\mathrm{a}}$ & & $31.2(26.1)$ & 11.2 \\
\hline TUN-15 & 100 & $14.0(12.7)$ & $17.0(15.6)$ & $30.2(28.6)$ & 10.7 \\
\hline
\end{tabular}

a The values in the parentheses were calculated from monomer and initiator content.

b $C_{\mathrm{d}}$ denotes the content of poly(styrene- $d_{8}$ ) determined by PGC.

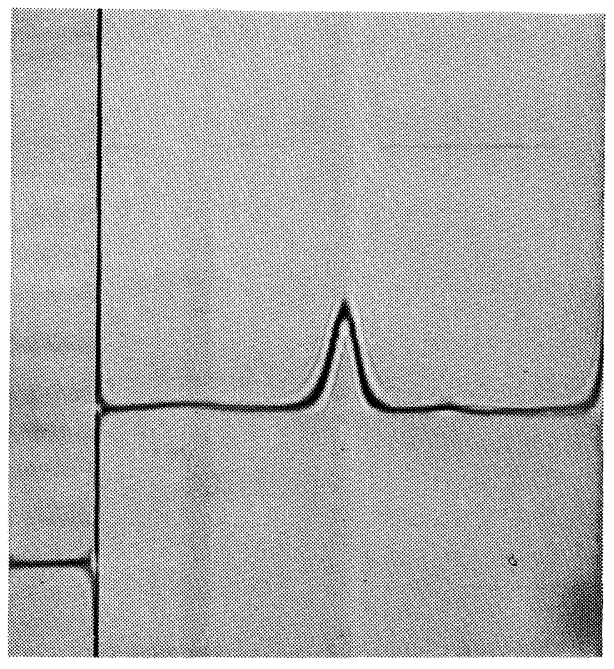

a

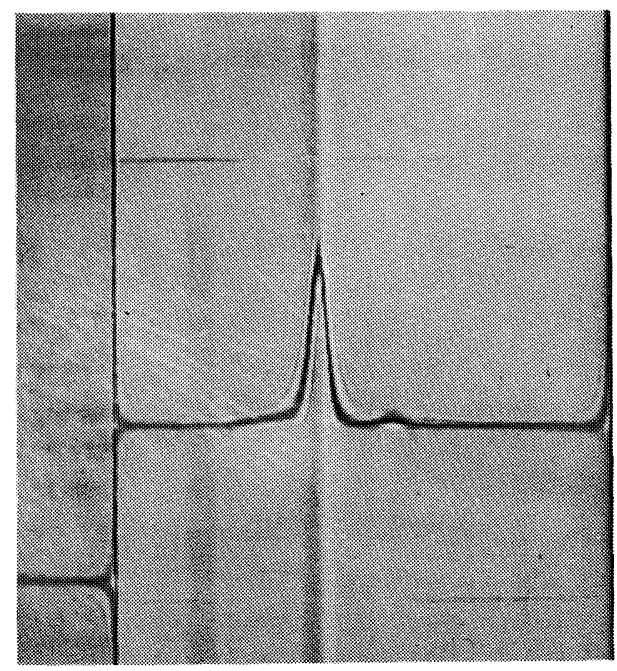

b

Figure 2. Schlieren patterns of unfractionated samples from Spinco Model E ultracentrifuge: solvent, cyclohexane; temperature, $35^{\circ} \mathrm{C}$; speed of rotation, $59780 \mathrm{rpm}$; angle, $75^{\circ}$.

(a) TUN-14; concn, $0.41 \mathrm{~g} \mathrm{dl}^{-1}$; time, $42 \mathrm{~min}$.

(b) TUN-15; concn, $0.41 \mathrm{~g} \mathrm{dl}^{-1}$; time, $42 \mathrm{~min}$.

two weeks. The amount of impurities on the lower side did not change after treatment with BP-Na. When the deuterated monomer thus purified was allowed to copolymerized with an ordinary styrene monomer, the polymerization of the deuterated monomer could be initiated by $s$-butyllithium or by polystyryl anions, but the propagation did not proceed satisfactorily. The color of poly(styrene- $d_{8}$ ) anion faded gradually.

Since ordinally fractional distillation methods ${ }^{1,6}$ were found to be ineffective for impurity removal, we used a fractional liquid chromatography to purify the monomer. The amount of impurity on the higher boiling point side diminished to less than $0.001 \mathrm{vol} \%$ as shown in Figure $1 \mathrm{~b}$. This amount of impurity was low enough to allow preparation of a sample with the desired molecular weight. The monomer thus purified was isolated and fractionally distilled in vacuo on a column (i.d. $8 \mathrm{~mm}, 300 \mathrm{~mm}$ long) packed with magnesium ribbons and treated with BP-Na just before use.

Two types of block copolymers, i.e., poly(styrene$d_{8}$ - $b$-styrene) (TUN-14) and poly(styrene- $b$-styrene$d_{8}$ - $b$-styrene) (TUN-15), were prepared using the deuterated monomers thus purified. The results are listed in Table I. The measured molecular weights of the precursors and block copolymer TUN-15 agreed with the values calculated from the amounts of initiator and monomers within experimental error. However, for TUN-14 the agree- 


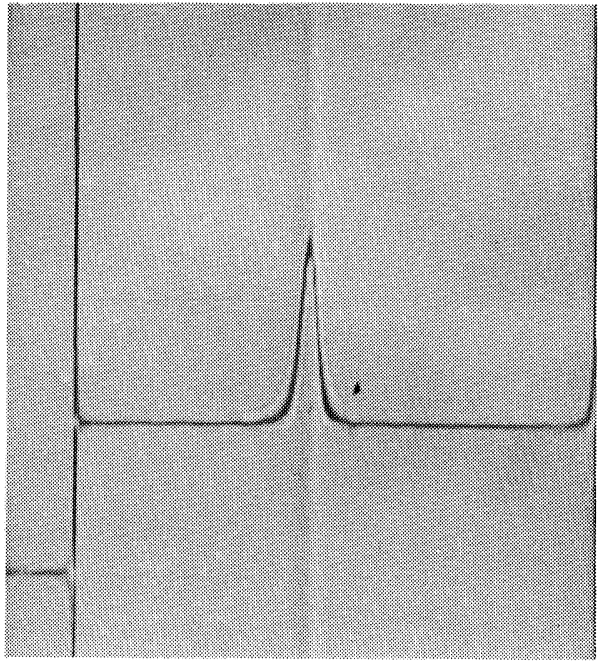

a

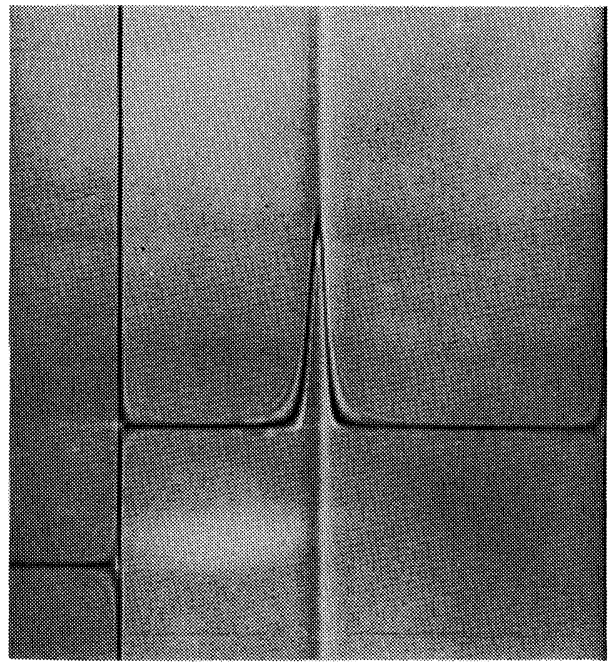

b

Figure 3. Schlieren patterns of fractionated samples.

(a) TUN-14; concn, $0.40 \mathrm{~g} \mathrm{dl}^{-1}$; time, $42 \mathrm{~min}$.

(b) TUN-15; concn, $0.38 \mathrm{~g} \mathrm{dl}^{-1}$; time, $41 \mathrm{~min}$.

Other experimental conditions are same as in the case of Figure 2.

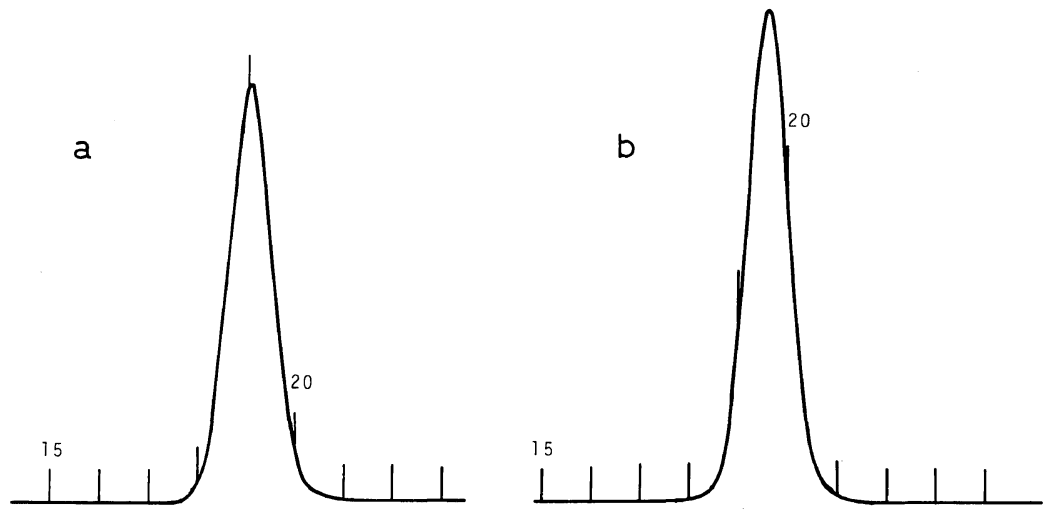

Figure 4. GPC chromatograms of fractionated block copolymers: (a) TUN-14; (b) TUN-15.

ment between calculated and observed values was not as satisfactory as that for TUN-15.

Figure 2 shows the schlieren patterns of TUN-14 and TUN-15. The main peaks are very sharp in both cases, but broad small peaks are found on both sides of the main peak for TUN-14 and a small peak on the higher molecular weight side for TUN-15. Moreover, a tail appears to exist on the lower molecular weight side for TUN-15.

The fact that the impurities responsible for these small peaks were removed by precipitational fractionation can be seen from Figure 3. The high purity and narrow molecular weight distributions of these samples were also checked with GPC chromatograms as shown in Figure 4. The molecular characteristics following fractionation are summarized in Table II. In calculating $M_{w}$ from the measurements of light scattering, the difference ${ }^{10}$ of $\partial n / \partial c$ between polystyrene and poly $\left(\right.$ styrene- $\left.d_{8}\right)$ in toluene was ignored, since the content of poly(styrene- $\left.d_{8}\right)$ was low. The measured values of $M_{w}$ were in good agreement with $M_{n}$. The polydispersity index $M_{w} / M_{n}$ was calculated from GPC data after being corrected for broadening effects with standards 
Table II. Molecular characteristics of fractionated block copolymers

\begin{tabular}{cccccc}
\hline $\begin{array}{c}\text { Sample } \\
\text { code }\end{array}$ & $\begin{array}{c}M_{n} \\
\times 10^{-4}\end{array}$ & $\begin{array}{c}M_{w} \\
\times 10^{-4}\end{array}$ & $\begin{array}{c}M_{w} / M_{n} \\
(\mathrm{GPC})\end{array}$ & $\begin{array}{c}C_{\mathrm{d}} \\
\%\end{array}$ & $\begin{array}{c}M_{w}(\mathrm{~d})^{\mathrm{a}} \\
\times 10^{-4}\end{array}$ \\
\hline TUN-14 & 41.6 & 42.6 & 1.04 & 6.3 & 2.7 \\
TUN-15 & 29.3 & 28.6 & 1.03 & 10.7 & 3.1 \\
\hline
\end{tabular}

${ }^{\text {a }} M_{w}(\mathrm{~d})$ are molecular weights of poly(styrene- $\left.d_{8}\right)$ blocks calculated from the equation $M_{w} \times\left(C_{\mathrm{d}} / 100\right)$.

polystyrenes.

Comparing the corresponding data in Tables I and II, we find that both the molecular weight and the content of poly(styrene- $\left.d_{8}\right)$ in TUN-15 before and after fractionation agree well with each other, but this is not the case with TUN-14. This fact suggests the existence of a deuterated homopolymer in the unfractionated TUN-14 and is consistent with the presence of a broad small peak on the lower molecular weight side of the schlieren pattern in Figure 2a. Thus, it is likely that the deuterated monomer used for TUN-14 contains impuritits higher than that for TUN-15. However, we note that this was not caused by the impurities contained originally in the monomer. Examination showed that the impurity was benzophenone sublimated during the distillation of the monomer, since we distilled the monomer completely and used the last fractions for the preparation of TUN-14.

Thus, the block copolymers of styrene and styrene- $d_{8}$ with narrow molecular weight distributions can be prepared provided the commercially available monomer is properly purified.

Acknowledgements. We should like to thank Mr. Y. Miyamoto and Mr. H. Funabashi at the
Technological University of Nagaoka for their kind help with the liquid chromatography and sample preparation, and $\mathrm{Mr}$. K. Endo of the same university for his assistance in the GPC experiments and Dr. R. Fleming of the National Bureau of Standards for helping with the neutron activation analysis.

This work was supported in part by a Grant-inAid for Scientific Research (No. 547087) from the Ministry of Education, Science and Culture of Japan.

\section{REFERENCES}

1. J. P. Cotton, D. Decker, H. Benoit, B. Farnoux, J. Higgins, G. Jannink, R. Ober, C. Picot, and J. des Cloizeaux, Macromolecules, 7, 863 (1974).

2. M. Daoud, J. P. Cotton, B. Farnoux, G. Jannink, G. Sarma, H. Benoit, R. Duplessix, C. Picot, and P. G. de Gennes, Macromolecules, 8, 804 (1975).

3. R. Duplessix, J. P. Cotton, H. Benoit, and C. Picot, Polymer, 20, 1181 (1979).

4. J. M. Guenet, C. Picot, and H. Benoit, Macromolecules, 12, 86 (1979).

5. M. Duval, R. Duplessix, C. Picot, D. Decker, P. Rempp, H. Benoit, J. P. Cotton, G. Jannink, B. Farnoux, and R. Ober, J. Polym. Sci., Polym. Lett. $E d$., 14, 585 (1976).

6. L. M. Ionescu, Doctor Thesis, L'Universite Louis Pasteur de Strasbourg, 1976.

7. D. de Soete, R. Gijbels, and J. Hoste, "Neutron Activation Analysis," Wiley-Interscience, New York, N.Y., 1972.

8. H. Ohtani, S. Tsuge, Y. Matsushita, and M. Nagasawa, Polym. J., 14, 495 (1982).

9. Y. Matsushita, H. Choshi, T. Fujimoto, and M. Nagasawa, Macromolecules, 13, 1053 (1980).

10. C. Strazielle, and H. Benoit, Macromolecules, 8, 203 (1975). 